\title{
Failure of target attainment of beta-lactam antibiotics in critically ill patients and associated risk factors: a two-center prospective study (EXPAT)
}

Alan Abdulla ${ }^{1 *}$ (D, Annemieke Dijkstra², Nicole G. M. Hunfeld ${ }^{1,3}$, Henrik Endeman ${ }^{3}$, Soma Bahmany ${ }^{1}$, Tim M. J. Ewoldt', Anouk E. Muller ${ }^{4,5}$, Teun van Gelder ${ }^{6}$, Diederik Gommers ${ }^{3}$ and Birgit C. P. Koch ${ }^{1}$

\begin{abstract}
Background: Early and appropriate antibiotic dosing is associated with improved clinical outcomes in critically ill patients, yet target attainment remains a challenge. Traditional antibiotic dosing is not suitable in critically ill patients, since these patients undergo physiological alterations that strongly affect antibiotic exposure. For betalactam antibiotics, the unbound plasma concentrations above at least one to four times the minimal inhibitory concentration (MIC) for 100\% of the dosing interval (100\% $\mathrm{fT}>1-4 \times \mathrm{MIC})$ have been proposed as pharmacodynamic targets (PDTs) to maximize bacteriological and clinical responses. The objectives of this study are to describe the PDT attainment in critically ill patients and to identify risk factors for target non-attainment.

Methods: This prospective observational study was performed in two ICUs in the Netherlands. We enrolled adult patients treated with the following beta-lactam antibiotics: amoxicillin (with or without clavulanic acid), cefotaxime, ceftazidime, ceftriaxone, cefuroxime, and meropenem. Based on five samples within a dosing interval at day 2 of therapy, the time unbound concentrations above the epidemiological cut-off ( $f \mathrm{~T}>\mathrm{MIC}_{\mathrm{ECOFF}}$ and $f \mathrm{~T}>4 \times \mathrm{MIC} \mathrm{E}_{\mathrm{ECOFF}}$ ) were determined. Secondary endpoints were estimated multivariate binomial and binary logistic regression models, for examining the association of PDT attainment with patient characteristics and clinical outcomes.

Results: A total of 147 patients were included, of whom 63.3\% achieved PDT of 100\% fT > MICECOFF and 36.7\% achieved 100\% fT $>4 \times \mathrm{MIC}_{\mathrm{ECOFF}}$. Regression analysis identified male gender, estimated glomerular filtration rate (eGFR) $\geq 90 \mathrm{~mL} / \mathrm{min} / 1.73 \mathrm{~m}^{2}$, and high body mass index (BMI) as risk factors for target non-attainment. Use of continuous renal replacement therapy (CRRT) and high serum urea significantly increased the probability of target attainment. In addition, we found a significant association between the $100 \% \mathrm{fT}>\mathrm{MIC}_{\mathrm{ECOFF}}$ target attainment and ICU length of stay (LOS), but no significant correlation was found for the 30-day survival.

(Continued on next page)
\end{abstract}

\footnotetext{
*Correspondence: a.abdulla@erasmusmc.nl

${ }^{1}$ Department of Hospital Pharmacy, Erasmus University Medical Center, P.O. Box 2040, 3000 CA Rotterdam, the Netherlands

Full list of author information is available at the end of the article
}

(C) The Author(s). 2020 Open Access This article is licensed under a Creative Commons Attribution 4.0 International License, which permits use, sharing, adaptation, distribution and reproduction in any medium or format, as long as you give appropriate credit to the original author(s) and the source, provide a link to the Creative Commons licence, and indicate if changes were made. The images or other third party material in this article are included in the article's Creative Commons licence, unless indicated otherwise in a credit line to the material. If material is not included in the article's Creative Commons licence and your intended use is not permitted by statutory regulation or exceeds the permitted use, you will need to obtain permission directly from the copyright holder. To view a copy of this licence, visit http://creativecommons.org/licenses/by/4.0/ The Creative Commons Public Domain Dedication waiver (http://creativecommons.org/publicdomain/zero/1.0/) applies to the data made available in this article, unless otherwise stated in a credit line to the data. 
(Continued from previous page)

Conclusions: Traditional beta-lactam dosing results in low target attainment in the majority of critically ill patients. Male gender, high BMI, and high eGFR were significant risk factors for target non-attainment. These predictors, together with therapeutic drug monitoring, may help ICU clinicians in optimizing beta-lactam dosing in critically ill patients.

Trial registration: Netherlands Trial Registry (EXPAT trial), NTR 5632. Registered on 7 December 2015.

Keywords: Beta-lactam, Critically ill patients, Pharmacokinetics, Pharmacodynamics, Target attainment, Risk factors

\section{Key messages}

- Less than two-thirds of our study population achieved the $100 \% \mathrm{fT}>\mathrm{MIC}_{\mathrm{ECOFF}}$ target, and only in one-third the $100 \% f \mathrm{~T}>4 \times \mathrm{MIC}_{\mathrm{ECOFF}}$ target was achieved.

- Male gender, high BMI, and eGFR $\geq 90 \mathrm{~mL} / \mathrm{min} /$ $1.73 \mathrm{~m}^{2}$ were significantly associated with target non-attainment, while the use of CRRT and high serum urea increased the probability of target attainment.

- For the $100 \% f \mathrm{~T}>\mathrm{MIC}_{\mathrm{ECOFF}}$ regression model, target non-attainment was significantly associated with the clinical outcome ICU length of stay, but no significant association was found for the 30-day survival.

\section{Introduction}

Large multicenter studies have reported antibiotic use in 64 to $71 \%$ of patients during their stay in the intensive care unit (ICU) [1, 2]. To adequately prevent and treat severe infections in critically ill patients, it is important that patients are treated with an appropriate dosing regimen of antibiotics [3-5]. However, dose-finding studies typically only include non-ICU populations. Various studies have shown that pathophysiological changes related to critical illness (i.e., altered fluid status, changes in serum albumin concentrations, renal and hepatic dysfunction, systemic inflammatory response syndrome, and microvascular failure) substantially change the pharmacokinetics (PK) and thereby the exposure to antibiotics $[6,7]$. Moreover, critically ill patients represent a highly heterogeneous population with a wide distribution of patients' ages, severities of illness, co-morbidities, source of infections, and outcomes [8]. These challenging conditions make it difficult to achieve optimal exposure in critically ill patients when using standard dosing regimens for beta-lactam antibiotics.

Beta-lactam antibiotics are amongst the most commonly used antibiotics in the ICU setting. These antibiotics exhibit time-dependent bacterial killing. Successful outcome is associated with the percentage of time $(\mathrm{T})$ of the dosing interval in which the unbound (free, $f$ ) serum antibiotic concentration remains above the minimum inhibitory concentration $(f \mathrm{~T}>\mathrm{MIC})$. For these antibiotics, the $f \mathrm{~T}>\mathrm{MIC}$ value needed for bactericidal activity is between 40 and $70 \%$ in in vivo infection models [9, 10], although clinical data suggests optimal efficacy is achieved at $100 \%$ $f \mathrm{~T}>\mathrm{MIC}$ in critically ill patients [10-12]. Patients achieving $100 \% f \mathrm{~T}>\mathrm{MIC}$ have significantly higher rates of clinical cure and bacteriological eradication [12-15]. To maximize the probability of clinical efficacy in critically ill patients, unbound plasma concentration from one up to four times the MIC for $100 \%$ of the dosing interval $(100 \% f \mathrm{~T}>1-4 \times \mathrm{MIC})$ has been identified as pharmacodynamic targets (PDTs) [1619]. Further increasing the exposure does not appear to increase the rate or extent of bacterial killing [20].

Target attainment is reported in only 40 to $60 \%$ of critically ill patients treated with beta-lactam antibiotics [21, 22]. That said, achieving the high ICU targets is not easy, particularly when conventional beta-lactam dosing regimens are used. Simply increasing the standard dosing for this group of antibiotics in all critically ill patients is not an optimal strategy, since high dosing regimens might result in trough levels associated with overexposure and toxicity [23]. Thus, it appears necessary to individualize betalactam dosing regimens in critically ill patients. Accordingly, identifying patients at risk could prompt clinicians to consider more individualized dosing regimens and regular therapeutic drug monitoring (TDM).

To our knowledge, only a few other studies have attempted to quantify patient characteristics as potential predictors for beta-lactam antibiotics target attainment in critically ill patients [14, 24-27]. In some of these studies, limited numbers of patients and/or different beta-lactam antibiotics were investigated, while in only two of these studies, target attainment and relevant factors associated with clinical outcomes were investigated $[24,27]$. However, the relationship between target attainment and the clinical outcomes ICU length of stay (LOS) and mortality has not yet been clarified. Therefore, the goals of this study are to determine the prevalence of target attainment of six frequently used betalactam antibiotics in ICUs in Europe and to identify risk factors and clinical outcomes associated with target nonattainment. 


\section{Methods}

\section{Study design}

This prospective, observational, two-center pharmacokinetics/pharmacodynamics (PK/PD) study was performed in the ICU departments of the Erasmus University Medical Center and Maasstad Hospital, Rotterdam, the Netherlands. The study protocol (EXPAT, NL53551.078.15) was approved by the Erasmus MC Medical Ethics Committee.

\section{Study population and size}

All patients admitted to the ICU between January 2016 and June 2017 and treated for a (presumed) infection with intravenous amoxicillin (with or without clavulanic acid), cefotaxime, ceftazidime, ceftriaxone, cefuroxime, or meropenem were assessed for inclusion. Eligible for enrollment were patients (1) aged $\geq 18$ years, (2) expected ICU stay $>72 \mathrm{~h}$, and (3) intravenous intermittent therapy of the study antibiotics. Initiation of study antibiotics, dosage, and duration of therapy were selected during a daily routine multidisciplinary consultation between the attending physician and an infectious disease specialist. Patients were excluded if (1) written informed consent was not obtained, (2) antibiotics were stopped before sampling, or (3) admitted to the ICU for burn injuries. Patient information was collected, including demographic data, clinical data, laboratory data, and antibiotic dosing data during hospitalization within the first 3 days after the start of the antibiotic therapy.

A formal sample size calculation was not needed because of the descriptive and noncomparative setting of this study. A priori in our protocol, it was likely that at least for four of the target antibiotics a minimum of 20 patients could be included based on prescribing data in our study sites. For the analysis of PDT attainment and associated clinical outcomes, a sample size of at least 140 was anticipated to be adequate [28].

\section{Sample collection and analysis}

On day 2 after the start of antimicrobial therapy, in total, five venous blood samples were collected at 15-30 min before the start of a dose (trough concentration, $C_{\text {min }}$ ), 15-30 min (peak concentration, $C_{\max }$ ), $1 \mathrm{~h}$ and $3 \mathrm{~h}$ after the end of infusion, and at 15-30 min before the start of the next dose (second $C_{\text {min }}$ ). The exact sampling times and the dosage administered were recorded. Blood samples were stored at $2-8^{\circ} \mathrm{C}$ directly after drawing to maintain the integrity and centrifuged at $3000 \mathrm{rpm}$ for 6 min within $24 \mathrm{~h}$ after collection. The plasma was transferred to cryo-vials for frozen storage $\left(-80^{\circ} \mathrm{C}\right)$ until analysis. Plasma concentrations were determined by a multi-analyte UPLC-MS/MS [29]. The method was comprehensively validated according to the Food and Drug Administration (FDA) guidance on bioanalytical method validation [30]. All observed concentrations were corrected for protein binding in critically ill patients, using average plasma protein binding (PPB) values [31]. Noncompartmental PK analysis of the plasma concentration-time data was performed using PKSolver (version 2.0) [32].

\section{Primary endpoints}

The PK/PD endpoints were the unbound concentration above the MIC at $100 \%$ (ICU target) of the dosing interval $(f \mathrm{~T}>\mathrm{MIC}$ and $f \mathrm{~T}>4 \times \mathrm{MIC})$. The percentage $f \mathrm{~T}>$ MIC was determined by calculating the intercept of the MIC values with the concentration-time curve. For each of the antibiotics, the epidemiological cut-off (ECOFF) of the presumed pathogens, i.e., the highest MIC for organisms devoid of phenotypically detectable acquired resistance mechanisms, as defined by the European Committee on Antimicrobial Susceptibility Testing (EUCAST), was used [33]. The following EUCAST epidemiological cut-off $\left(\mathrm{MIC}_{\mathrm{ECOFF}}\right)$ values were used: amoxicillin $8 \mathrm{mg} / \mathrm{L}$ (Enterobacterales), cefotaxime $4 \mathrm{mg} /$ L (Staphylococcus aureus), ceftazidime $8 \mathrm{mg} / \mathrm{L}$ (Pseudomonas aeruginosa), ceftriaxone $0.5 \mathrm{mg} / \mathrm{L}$ (Enterobacterales), cefuroxime $8 \mathrm{mg} / \mathrm{L}$ (Escherichia coli), and meropenem $2 \mathrm{mg} / \mathrm{L}$ (Pseudomonas aeruginosa). To assess the suitability of the empirical fixed dosing regimens considering $f \mathrm{~T}>\mathrm{MIC}$ and $f \mathrm{~T}>4 \times \mathrm{MIC}$, a MIC distribution of $0.03125-128 \mathrm{mg} / \mathrm{L}$ was tested for target attainment for each of the antibiotics.

\section{Secondary endpoints}

We defined ICU length of stay (LOS) and 30-day survival from the start of therapy (enrollment) as our secondary endpoints. Factors likely to contribute to these two outcomes were analyzed for association based on clinical relevancy and previously described relationships [14, 24-27]. These included patient characteristics (age, gender, body mass index (BMI)), illness severity score (Sequential Organ Failure Assessment (SOFA) score at the start of target antibiotic), serum albumin, serum urea, sepsis, estimated glomerular filtration rate (eGFR $\geq 90 \mathrm{~mL} / \mathrm{min} / 1.73 \mathrm{~m}^{2}$ ), and presence of continuous renal replacement therapy (CRRT).

\section{Statistical analysis}

All statistical analyses were performed using IBM-SPSS (version 24.0, IBM Corp., New York, NY, USA) and R software (version 3.3.3, R Project for Statistical Computing). Normality was assessed using the Shapiro-Wilk test. We analyzed our data using the following three steps. First, categorical variables were expressed as frequencies (percentages), and continuous variables were described as median values with the interquartile range (IQR; 25-75th percentile). Differences in categorical 
variables were calculated using Pearson chi-square test or Fisher's exact test as appropriate. The Mann-Whitney $U$ test was used to compare continuous variables.

For our primary outcome, i.e., PDT attainment, we estimated multivariate binary logistic regression analyses and present the odds ratios (ORs) and 95\% confidence intervals $(95 \% \mathrm{CI})$. We included SOFA score at inclusion in the multivariate analysis to control for all our regressions for clinically and relevant baseline characteristics.

For our secondary outcomes, we estimated multivariate negative binomial regression and binary logistic regression models examining the association of PDT attainment with ICU LOS and 30-day survival, respectively. For these regressions, we present the ORs and 95\% CI. Statistical significance was accepted at $p \leq 0.05$.

\section{Results}

Overall, a total of 147 patients were included in the study, and 712 serum samples were analyzed. Baseline patient demographic and clinical characteristics, stratified by the target attainment, are summarized in Table 1 . The median patient age was 63 years, and $61 \%$ of the patients were male. The median ICU LOS was 9 days, and 30-day all-cause mortality rate was $19.7 \%$.

\section{Pharmacokinetic parameters}

Box-and-whisker plots of unbound trough $\left(f C_{\min }\right)$ plasma concentrations observed for the different antibiotics are shown in Fig. 1, and peak $\left(f C_{\max }\right)$ plasma concentration plots can be found in Additional file 1. Large inter-patient variability was observed in the plasma concentration of the various antibiotics, e.g., a $f C_{\min }$ of cefotaxime ranging from 0.14 to $26 \mathrm{mg} / \mathrm{L}$. Detailed data describing the pooled antibiotic dosing and PK/PD indices are provided in Additional file 2.

\section{Target attainment}

The proportions of patients achieving the PDT of $100 \%$ $f \mathrm{~T}>\mathrm{MIC}_{\mathrm{ECOFF}}$ and $100 \% f \mathrm{~T}>4 \times \mathrm{MIC}_{\mathrm{ECOFF}}$ were $63.3 \%$ (93/147) and 36.7\% (44/147), respectively (Table 1 and Additional file 2). Compared to patients who did attain the target, younger male patients with higher creatinine clearance, higher serum albumin, higher white blood cell count, higher length, and lower urea and those who received concomitant antibiotics were more likely not to achieve the PDT. Moreover, $45.7 \%(21 / 46)$ and $31 \%(16 /$ 51) of those with an eGFR $\geq 90 \mathrm{~mL} / \mathrm{min} / 1.73 \mathrm{~m}^{2}$ at baseline and day 2 achieved the $100 \% f \mathrm{~T}>\mathrm{MIC}_{\mathrm{ECOFF}}$ target, respectively. Of all patients, twenty-eight (19\%) patients were treated with CRRT. Although not statistically significant, there was a noticeable clinically significant difference in the number of patients treated with CRRT between the groups who did and did not achieve the PDT, respectively $23.7 \%$ and $12.1 \%(p=0.06)$. The rates for $100 \% f \mathrm{~T}>\mathrm{MIC}_{\mathrm{ECOFF}}$ target attainment for amoxicillin, cefotaxime, ceftazidime, ceftriaxone, cefuroxime, and meropenem were 44.4\%, 57.0\%, 100\%, 94.1\%, 0\%, and $71.4 \%$, respectively (Additional file 2). Probability of reaching the target $100 \% f \mathrm{~T}>4 \times \mathrm{MIC}_{\mathrm{ECOFF}}$ was less than $25 \%$ for amoxicillin, cefotaxime, and cefuroxime, suggesting that for all these drugs, inadequate drug concentrations are obtained for pathogens with high MICs. Target attainment for various beta-lactam antibiotics and dosing regimens to reach the PDTs of $100 \% f \mathrm{~T}>\mathrm{MIC}$ and $100 \%$ $f \mathrm{~T}>4 \times \mathrm{MIC}$ for a range of MICs $(0.03125$ to $128 \mathrm{mg} / \mathrm{L})$ are shown in Fig. 2.

\section{Predictors for target (non-)attainment}

Predictor variables for target attainment were investigated in the multivariate binary logistic regression (Table 2). Variables associated with $100 \% f \mathrm{~T}>\mathrm{MIC}_{\mathrm{ECOFF}}$ target attainment include treatment with CRRT (OR 6.54, 95\% CI 1.47-48.61) and high serum urea (OR 1.09, 95\% CI 1.031.17). Male gender, on the other hand, was found to be significantly correlated with target non-attainment (OR 0.32 , 95\% CI 0.12-0.81). For the PDT $100 \% f \mathrm{~T}>$ $4 \times \mathrm{MIC}_{\mathrm{ECOFF}}$, target non-attainment was significantly more observed in patients with baseline eGFR $\geq 90 \mathrm{~mL} /$ $\mathrm{min} / 1.73 \mathrm{~m}^{2}$ (OR 0.14, 95\% CI 0.03-0.49) and high BMI (OR 0.91, 95\% CI 0.83-0.99). A high serum urea demonstrated a significant association (OR 1.05, 95\% CI 1.001.10) with $100 \% f \mathrm{~T}>4 \times \mathrm{MIC}$ target attainment.

\section{Predictors for clinical outcomes}

Table 3 shows the estimates for the multivariate binomial regression and binary logistic regression models examining the association of target attainment with ICU LOS and 30-day survival, respectively. The PDT of $100 \%$ $f \mathrm{~T}>\mathrm{MIC}_{\mathrm{ECOFF}}$ was significantly associated with the clinical outcome ICU LOS (OR 1.66, 95\% CI 1.19-2.32). In the multivariate models, the presence of CRRT was for both PDTs significantly associated with higher ICU LOS (OR, 2.08 [95\% CI, 1.38-3.20] and OR, 2.13 [95\% CI, 1.39-3.34], respectively). Furthermore, an eGFR $\geq 90$ $\mathrm{mL} / \mathrm{min} / 1.73 \mathrm{~m}^{2}$ was associated with increased ICU LOS in both PDT models (OR, 1.67 [95\% CI, 1.13-2.47] and OR, 1.69 [95\% CI, 1.12-2.56], respectively). Finally, there was no significant association for both models with the 30-day survival outcome (OR, 0.56 [95\% CI, 0.19-1.66] and OR, 1.24 [95\% CI, 0.44-3.73], respectively).

\section{Discussion}

In this prospective study, we describe detailed target attainment of six frequently used beta-lactam antibiotics and risk factors for target non-attainment in critically ill patients. Achievement of PK/PD targets was highly variable in the beta-lactam antibiotics analyzed in this study (Fig. 1), and the $100 \% f \mathrm{~T}>\mathrm{MIC}_{\mathrm{ECOFF}}$ target was 
Table 1 Baseline demographic characteristics, clinical data, PK/PD indices, and clinical outcomes of all patients included and between PDT attainment and non-attainment groups

\begin{tabular}{|c|c|c|c|c|}
\hline Characteristics & All patients $(n=147)$ & PDT attainment $(n=93)$ & $\begin{array}{l}\text { PDT non-attainment } \\
(n=54)\end{array}$ & $p$ value $^{a}$ \\
\hline \multicolumn{5}{|l|}{ Demographic data } \\
\hline Age (years) & $63(56-70)$ & $65.0(58.5-73.0)$ & $60.5(51.0-66.0)$ & 0.001 \\
\hline Sex (male/female) & $91 / 56$ & $51 / 42$ & $40 / 14$ & 0.021 \\
\hline Length (cm) & $172.2(10.7)$ & $169.7(10.1)$ & $176.6(10.3)$ & $<0.001$ \\
\hline Weight (kg) & 77 (70-90) & $75(68-90)$ & $80(70-90)$ & 0.339 \\
\hline $\mathrm{BMI}$ & $26.1(22.9-29.3)$ & $26.9(23.9-29.6)$ & $25.3(22.2-28.1)$ & 0.087 \\
\hline Concomitant antibiotics & & & & 0.029 \\
\hline No & $54(36.7 \%)$ & $28(30.1 \%)$ & $26(48.1 \%)$ & \\
\hline Yes $^{b}$ & $93(63.3 \%)$ & $65(69.9 \%)$ & $28(51.9 \%)$ & \\
\hline \multicolumn{5}{|l|}{ Clinical data at inclusion } \\
\hline SOFA & $11.0[7.0-15.0]$ & & & 0.293 \\
\hline $0-6$ & $28(19.0 \%)$ & $15(16.3 \%)$ & $13(24.1 \%)$ & \\
\hline $7-9$ & $32(21.8 \%)$ & $18(19.6 \%)$ & $14(25.9 \%)$ & \\
\hline $10-14$ & $38(25.9 \%)$ & $24(26.1 \%)$ & $14(25.9 \%)$ & \\
\hline 15 & $48(32.7 \%)$ & $35(38.0 \%)$ & $13(24.1 \%)$ & \\
\hline APACHE $\|$ & $23[18-27]$ & & & 0.161 \\
\hline $0-9$ & $5(3.4 \%)$ & $3(3.3 \%)$ & $2(3.7 \%)$ & \\
\hline $10-19$ & $39(26.7 \%)$ & $20(21.7 \%)$ & 19 (35.2\%) & \\
\hline $20-29$ & $85(58.2 \%)$ & $55(59.8 \%)$ & $30(55.6 \%)$ & \\
\hline$\geq 30$ & $17(11.6 \%)$ & $14(15.2 \%)$ & $3(5.6 \%)$ & \\
\hline Albumin $(g / L)$ & $26.3(7.3)$ & $24.9(7.0)$ & $28.5(7.3)$ & 0.003 \\
\hline Serum creatinine $(\mu \mathrm{mol} / \mathrm{L})$ & 102 [67-155] & $124.0[79.5-182.5]$ & 79.5 [56.8-106.3] & $<0.001$ \\
\hline Temperature $\left({ }^{\circ} \mathrm{C}\right)$ & $36.9[36.1-37.4]$ & $36.7[36.0-37.3]$ & $37.0[36.3-37.6]$ & 0.112 \\
\hline $\mathrm{WBC}\left(\times 10^{9} / \mathrm{L}\right)$ & $13.2[8.7-18.2]$ & $11.7[7.3-17.8]$ & $15.8[10.7-20.2]$ & 0.022 \\
\hline CRP $(\mathrm{mg} / \mathrm{L})$ & $111[35-226]$ & $120[46-242]$ & $91[15-175]$ & 0.072 \\
\hline Serum urea $(\mathrm{mmol} / \mathrm{L})$ & $8.9[6.1-16.5]$ & $12.4[7.1-19.2]$ & $6.6[4.8-9.3]$ & $<0.001$ \\
\hline eGFR (mL/min/1.73 m²) & & & & $<0.001$ \\
\hline$<30$ & $29(19.7 \%)$ & $25(26.9 \%)$ & $4(7.4 \%)$ & \\
\hline $30-50$ & $31(21.1 \%)$ & $28(30.1 \%)$ & $3(5.6 \%)$ & \\
\hline $50-90$ & $41(27.9 \%)$ & $19(20.4 \%)$ & $22(40.7 \%)$ & \\
\hline$>90$ & $46(31.3 \%)$ & $21(22.6 \%)$ & $25(46.3 \%)$ & \\
\hline CRRT & & & & 0.063 \\
\hline No & $119(81 \%)$ & $71(76.3 \%)$ & $48(88.9 \%)$ & \\
\hline Yes & $28(19 \%)$ & $22(23.7 \%)$ & $6(12.1 \%)$ & \\
\hline \multicolumn{5}{|l|}{ PK/PD indices } \\
\hline$\% f \mathrm{~T}>\mathrm{MIC}_{\mathrm{ECOFF}}$ & $84.2 \%$ & & & \\
\hline$\% f \mathrm{~T}>4 \times M I C_{E C O F F}$ & $51.7 \%$ & & & \\
\hline \multicolumn{5}{|l|}{ Clinical outcomes } \\
\hline ICU LOS (days) & 9 [4-15] & $11[6-20]$ & $5[3-12.8]$ & 0.005 \\
\hline 30-day mortality & $29(19.7 \%)$ & $22(24.2 \%)$ & $7(13.2 \%)$ & 0.135 \\
\hline
\end{tabular}

Values are presented as numbers (\%), median [25\%-75\% interquartile range], or mean ( \pm standard deviation). The numbers in bold are statistically significant APACHE II Acute Physiology and Chronic Health Evaluation II, BMI body mass index, CRP C-reactive protein, CRRT continuous renal replacement therapy, ECOFF epidemiological cut-off value, eGFR estimated glomerular filtration rate, calculated with the CKD-EPI Creatinine Equation, $f T>M I C$ the unbound concentrations above the minimum inhibitory concentration, ICU LOS intensive care unit length of stay, calculated from the start of study antibiotic until ICU discharge, PDT pharmacodynamic target, SOFA score Sequential Organ Failure Assessment score, WBC white blood cell count

${ }^{\text {a The }} p$ value between target attainment versus non-attainment patient population and the value in bold indicates a significant difference between the two groups $(p \leq 0.05)$

${ }^{\mathrm{b}}$ One or more additional antibiotics 


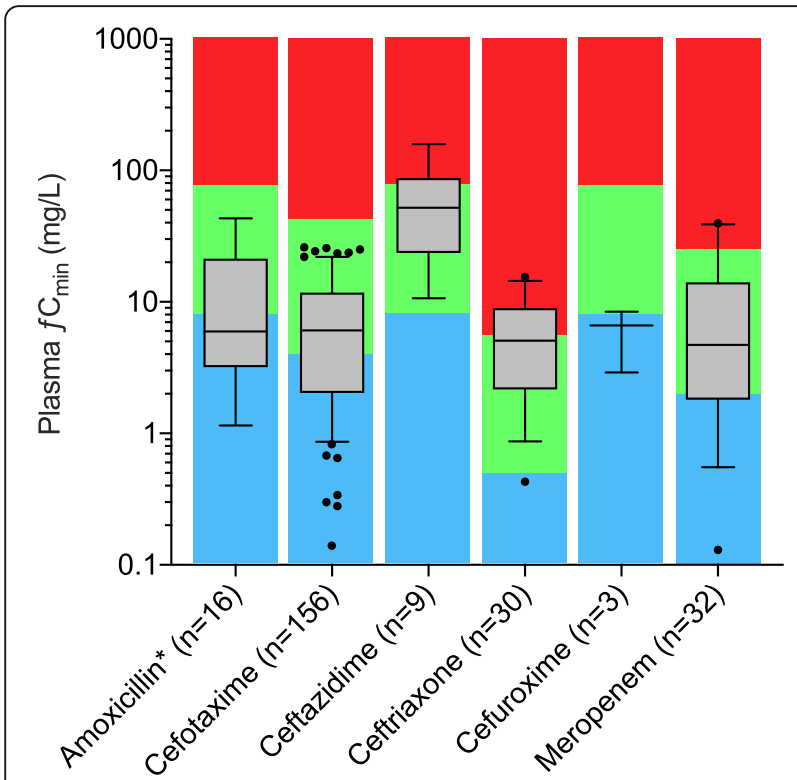

Fig. 1 Box (median, 25th and 75th percentiles) and whisker (10th and 90th percentiles) plots of unbound trough $\left(f C_{\text {min }}\right)$ plasma concentrations observed in critically ill patients treated with beta-lactam antibiotics. The green areas indicate the target exposure $\left(f C_{\min }=1-10 \times M I C_{\text {ECOFF }}\right)$, the blue areas indicate suboptimal exposure $\left(f C_{\min }<1 \times M \mid C_{E C O F F}\right)$, and the red areas indicate threshold for dose reduction $\left(f C_{\min }>10 \times M I C_{E C O F F}\right)$. The numbers of trough samples $(n)$ are presented per antibiotic. Outliers are removed using the ROUT method $(Q=0.5 \%)$. Filled circles are remaining outliers. *Amoxicillin and amoxicillin/clavulanic acid

achieved in $63.3 \%$ of the study population. We identified male gender, high BMI, and high eGFR as risk target non-attainment in our study population. Moreover, we found a significant association between the $100 \% \mathrm{f} \mathrm{T}>$ $\mathrm{MIC}_{\mathrm{ECOFF}}$ target attainment and ICU LOS, but no significant correlation was found for the 30-day survival.

Roberts et al. found that critically ill patients failing to attain even the most conservative beta-lactam exposure target of $50 \% f \mathrm{~T}>\mathrm{MIC}$ were $32 \%$ less likely to have a positive clinical outcome (defined as completion of treatment course without change or addition of antibiotic therapy) [21]. In addition, they found an association between positive clinical outcome and an increasing 100\% $f \mathrm{~T}>\mathrm{MIC}$ ratio (OR 1.53, $p=0.03$ ) [21]. Considering that critically ill patients are vulnerable to suboptimal dosing and represent a source of selection of resistance to antibiotics, we also assessed the probability of $100 \% f \mathrm{~T}>$ $4 \times \mathrm{MIC}_{\mathrm{ECOFF}}$ target attainment, as this would allow for the maximal bacterial killing and also protect against bacterial regrowth [34-36]. In our study, the $100 \% f \mathrm{~T}$ > $4 \times \mathrm{MIC}_{\mathrm{ECOFF}}$ target was achieved in only $36.7 \%$ of the patients.

Antimicrobial dosing in critically ill patients requires consideration of drug distribution and clearance in the setting of end-organ failure, fluctuations in fluid status, and drug interactions. However, the findings of our study suggest that target attainment during beta-lactam therapy in critically ill patients may be anticipated at the bedside prior to antibiotic initiation. Predictor variables in the multivariate analysis associated with increased odds for target non-attainment were male gender and eGFR (Table 2). These associations in critically ill patients treated with beta-lactam antibiotics are in line with previous studies [25-27].

The observed effect of gender on drug exposure can be explained by the fact that, on average, greater volume of distribution (plasma volume and intra-/extracellular water) and higher drug clearance is observed in men [37]. Although gender is easy to implement in risk factor models for target non-attainment, future studies should be designed with a primary focus on this topic to better understand of the basic mechanisms of gender differences and the implications for clinical management [37].

In our study population, the probability of the $100 \% f \mathrm{~T}>4 \times \mathrm{MIC}$ target non-attainment was significantly associated with an eGFR $\geq 90 \mathrm{~mL} / \mathrm{min} / 1.73 \mathrm{~m}^{2}$ (OR 0.14, 95\% CI 0.03-0.49) at inclusion. eGFR calculated on serum creatinine is the best surrogate marker of renal clearance. In total, $31.3 \%$ of our population had an eGFR $\geq 90 \mathrm{~mL} / \mathrm{min} / 1.73 \mathrm{~m}^{2}$, while $46.3 \%$ in the non-attainment group did so (Table 2). This means that patients with presumed "normal" or elevated renal function are at risk of target non-attainment and need to be identified early so that appropriate dose adjustment can be made. Moreover, Imani et al. assessed the performance of eGFR as an independent predictor for target non-attainment using a ROC curve and found an eGFR threshold value of $\geq 71.5 \mathrm{~mL} / \mathrm{min} / 1.73 \mathrm{~m}^{2}$ had a sensitivity and specificity of $77 \%$ and $65 \%$, respectively [26]. As beta-lactam antibiotics are predominantly cleared by the kidney, high renal function (eGFR $\geq 130 \mathrm{~mL} / \mathrm{min}$ ), as observed in augmented renal clearance (ARC), contributes even more to suboptimal PK/PD target attainment. ARC is observed in $30-65 \%$ of patients during the first week in the ICU $[14,38,39]$, and both age and male gender are independently associated with ARC [39]. Moreover, ARC is a strong predictor for one or more undetectable trough concentrations [OR 3.3, 95\% CI 1.11-9.94] [14]. Carrié et al. reported that eGFR $\geq 170 \mathrm{~mL} / \mathrm{min}$ were significantly associated with $\mathrm{T}<4 \times \mathrm{MIC}$ [OR 10.1; (2.4-41.6); $p=0.001$ ] [24]. The ability to rapidly predict the risk of target non-attainment in patients with ARC using available eGFR has considerable clinical value.

Furthermore, in the multivariate analysis, we found a strong and significant association between target attainment and treatment with CRRT. To our knowledge, this is the only study where in a multivariate analysis this association was demonstrated. Not surprisingly, considering that beta-lactams are predominantly cleared via 


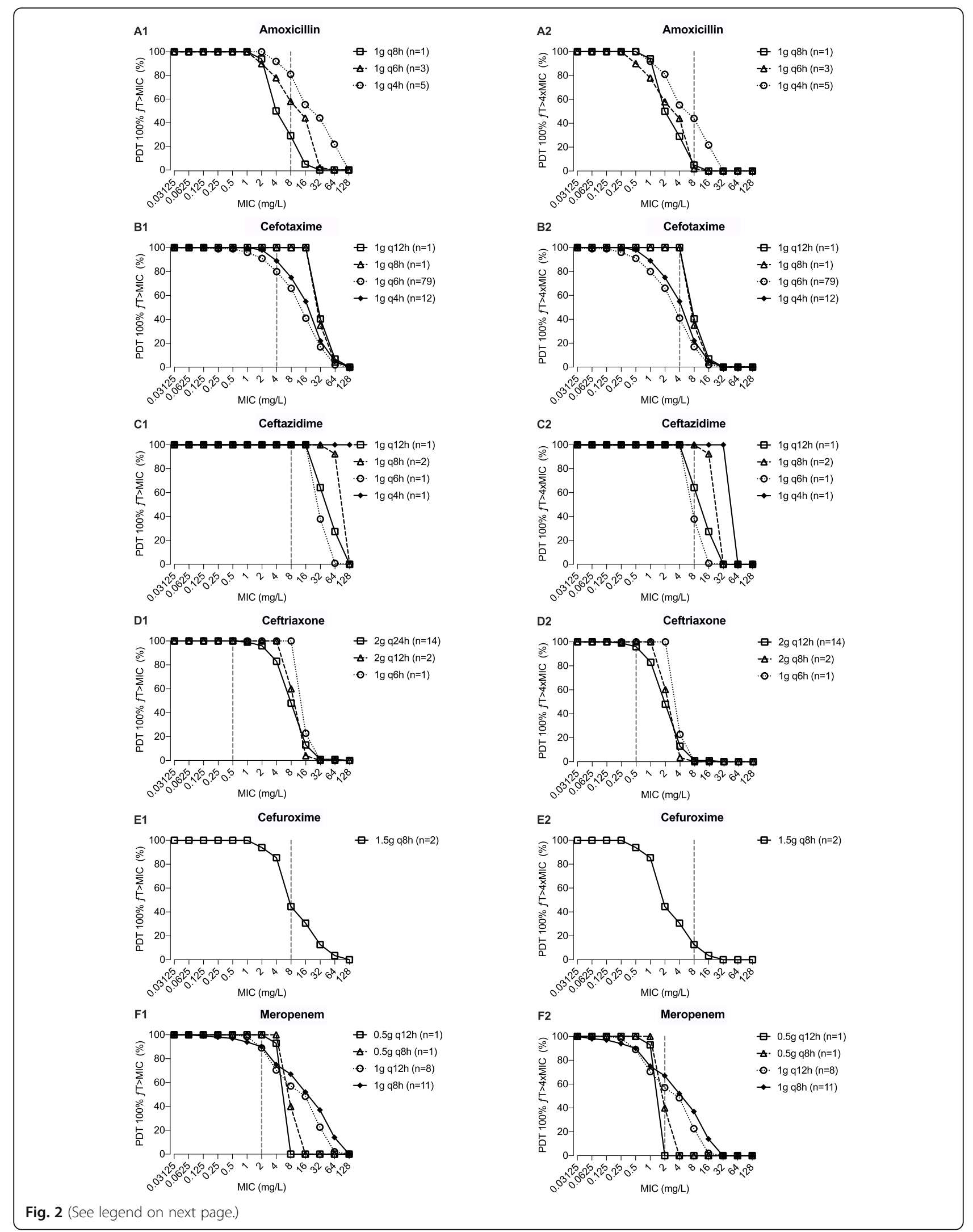


(See figure on previous page.)

Fig. 2 Target attainment in ICU patients for various beta-lactams and dosing regimens to reach the PDTs $100 \% f \mathrm{f}>\mathrm{MIC}(\mathbf{A} 1-\mathbf{F} \mathbf{1})$ and $100 \% \mathrm{fT}>$ 4XMIC (A2-F2) for a range of MICs. The numbers of patients ( $n$ ) are presented per antibiotic and dose regimen. The dotted horizontal line indicates the intercept with the EUCAST epidemiological cut-off (ECOFF) breakpoints: amoxicillin $8 \mathrm{mg} / \mathrm{L}$ (Enterobacterales), cefotaxime $4 \mathrm{mg} / \mathrm{L}$ (Staphylococcus aureus), ceftazidime $8 \mathrm{mg} / \mathrm{L}$ (Pseudomonas aeruginosa), ceftriaxone $0.5 \mathrm{mg} / \mathrm{L}$ (Enterobacterales), cefuroxime $8 \mathrm{mg} / \mathrm{L}$ (Escherichia coli), and meropenem $2 \mathrm{mg} / \mathrm{L}$ (Pseudomonas aeruginosa)

renal elimination. At the same time, these patients may be at risk for overexposure and toxicity due to the reduced elimination. However, predicting beta-lactam concentrations during treatment with CRRT is challenging, as both volume of distribution and total drug clearance are affected, and both parameters may be significantly disturbed during critical illness. In addition, it is important to realize that the effect of CRRT on target attainment may be unpredictably affected by for example the type of membrane, device settings, and intensity [40].

Evidence regarding relevant predictor variables with clinical outcomes in critically ill patients are still limited. Huttner et al. found that ARC was associated with undetectable beta-lactam antibiotic trough concentrations, but failed to demonstrate a link between ARC or low beta-lactam trough concentrations with clinical failure [14]. In contrast, Carrié et al. reported that beta-lactam underexposure was associated with higher rates of therapeutic failure in septic critically ill patients [24]. In our population, we found a significant association for the $100 \% f \mathrm{~T}>\mathrm{MIC}_{\mathrm{ECOFF}}$ PDT model with the clinical outcome ICU LOS. The link with this outcome is a new finding. Interestingly, the ICU LOS increases as $100 \%$ $f \mathrm{~T}>\mathrm{MIC}$ is achieved. This is also reflected in the

Table 2 Multivariate binary logistic regression in ICU patients, analysis predicting attainment achieving PDT of (A) 100\% fT > MIC and (B) 100\% fT > 4XMIC as the dependent factor

\begin{tabular}{|c|c|c|}
\hline Predictor variables & $\begin{array}{l}100 \% \mathrm{fT}>\mathrm{MIC} \\
\text { OR }(95 \% \mathrm{Cl})\end{array}$ & $\begin{array}{l}100 \% f \mathrm{~T}>4 \times \mathrm{MIC} \\
\text { OR }(95 \% \mathrm{Cl})\end{array}$ \\
\hline Male gender & $0.32(0.12-0.81)$ & $0.60(0.23-1.51)$ \\
\hline Age (years) & $1.03(0.99-1.07)$ & $0.98(0.94-1.01)$ \\
\hline BMI $\left(\mathrm{mg} / \mathrm{kg}^{2}\right)$ & $0.98(0.91-1.05)$ & $0.91(0.83-0.99)$ \\
\hline Serum urea (mmol/L) & $1.09(1.03-1.17)$ & $1.05(1.00-1.10)$ \\
\hline $\mathrm{eGFR} \geq 90\left(\mathrm{~mL} / \mathrm{min} / 1.73 \mathrm{~m}^{2}\right)$ & $0.69(0.25-1.94)$ & $0.14(0.03-0.49)$ \\
\hline SOFA score & $1.05(0.96-1.16)$ & $0.95(0.85-1.05)$ \\
\hline CRRT & $6.54(1.47-48.61)$ & $2.26(0.73-6.97)$ \\
\hline Sepsis & $1.18(0.38-3.88)$ & $1.31(0.43-3.88)$ \\
\hline
\end{tabular}

The estimates are odds ratios (ORs) and $95 \%$ confidence intervals. The numbers in bold are statistically significant. Statistical significance was accepted at $p \leq 0.05$. McFadden $R$-squared for models $\mathrm{A}$ and $\mathrm{B}$ are 0.21 and 0.18 , respectively, representing good fit

$B M I$ body mass index, CRRT continuous renal replacement therapy, eGFR estimated glomerular filtration rate calculated with the CKD-EPI Creatinine Equation, $f T>M I C$ the unbound concentrations above the minimum inhibitory concentration, PDT pharmacodynamic target, SOFA score Sequential Organ Failure Assessment score significant difference of the median admission days in both groups (Table 1). An explanation for the target attainment association with ICU LOS may be the fact that the elderly and the most ill patients in our population stayed longer at the ICU. These patients have relatively worse end-organ functions (including renal clearance) and therefore have higher exposure and are more ontarget. Patients who died could have had an effect on the outcomes of the predictive LOS models. Therefore, we also performed the binomial regression models while excluding the non-survivors (data not presented). However, this had no effect on the significant variables found in both PDT models. Furthermore, in both PDT models, CRRT was significantly associated with higher ICU LOS. In addition, eGFR $\geq 90 \mathrm{~mL} / \mathrm{min} / 1.73 \mathrm{~m}^{2}$ was independently and convincingly associated with increased ICU LOS in both PDT models (Table 3). It should be noted that patients receiving CRRT $(n=28)$ at any time during the antibiotic therapy were not excluded from this analysis. However, an eGFR $\geq 90 \mathrm{~mL} / \mathrm{min} / 1.73 \mathrm{~m}^{2}$ was still independently associated with increased ICU LOS when patients on CRRT are excluded (data not shown). Although increased renal clearance and ARC is highly prevalent in critically ill patients, in practice, clinicians may fail to address this as a risk factor and prescribe standard beta-lactam dosing. Indeed, the complexity and dynamic nature of critically ill patients and the heterogeneity in their pharmacokinetic response make associations of clinical variables and the calculated risk of target non-attainment of beta-lactam antibiotics difficult to apply without supporting tools.

Therapeutic drug monitoring (TDM) combined with population PK models with appropriate co-variables can be used to interpret the complex PK in critically ill patients and to support in optimizing individual dosing to improve predefined targets [41-43]. However, the lack of guidelines, long turnaround times, and limited access to beta-lactam TDM services are potential barriers to its implementation [44]. Routine TDM with same-day antibiotic dose adaptation using immunoassays over the more commonly used chromatographic methods could contribute to overcoming these barriers in routine clinical practice [45]. However, pending a large randomized trial investigating the effect of TDM of beta-lactam antibiotics on clinical outcome in critically ill patients [46], the clinical impact on patient's prognosis using this strategy is not yet fully demonstrated. In view of the fact 
Table 3 Multivariate regression models in ICU patients for PDT attainment and odds ratio estimates for the association with the clinical outcomes (A) ICU LOS and (B) 30-day survival

\begin{tabular}{|c|c|c|}
\hline Models and variables & $\begin{array}{l}\text { ICU LOS } \\
\text { OR }(95 \% \text { CI) }\end{array}$ & $\begin{array}{l}\text { 30-day survival } \\
\text { OR }(95 \% \mathrm{Cl})\end{array}$ \\
\hline Regression model, PDT: $100 \%$ fT > MIC & $1.66(1.19-2.32)$ & $0.58(0.19-1.66)$ \\
\hline Age (years) & $0.99(0.98-1.01)$ & $1.02(0.98-1.05)$ \\
\hline CRRT & $2.08(1.38-3.20)$ & $0.41(0.13-1.33)$ \\
\hline Sepsis & $0.91(0.61-1.37)$ & $0.90(0.30-2.86)$ \\
\hline Serum urea (mmol/L) & $1.00(0.99-1.02)$ & $1.05(0.99-1.11)$ \\
\hline SOFA score & $1.00(0.96-1.03)$ & $0.95(0.85-1.05)$ \\
\hline $\mathrm{eGFR} \geq 90\left(\mathrm{~mL} / \mathrm{min} / 1.73 \mathrm{~m}^{2}\right)$ & $1.67(1.13-2.47)$ & $1.97(0.66-6.88)$ \\
\hline Regression model, PDT: $100 \% \mathrm{fT}>4 \times \mathrm{MIC}$ & $1.26(0.88-1.82)$ & $1.24(0.44-3.73)$ \\
\hline Age (years) & $1.00(0.98-1.01)$ & $1.01(0.98-1.05)$ \\
\hline CRRT & $2.13(1.39-3.34)$ & $0.35(0.11-1.11)$ \\
\hline Sepsis & $0.89(0.59-1.34)$ & $0.91(0.31-2.88)$ \\
\hline Serum urea (mmol/L) & $1.01(0.99-1.03)$ & $1.04(0.98-1.10)$ \\
\hline SOFA score & $1.01(0.97-1.05)$ & $0.94(0.84-1.05)$ \\
\hline eGFR $\geq 90\left(\mathrm{~mL} / \mathrm{min} / 1.73 \mathrm{~m}^{2}\right)$ & $1.69(1.12-2.56)$ & $2.09(0.66-7.22)$ \\
\hline
\end{tabular}

The estimates are odds ratios (ORs) and $95 \%$ confidence intervals. The numbers in bold are statistically significant. Statistical significance was accepted at $p \leq 0.05$ CRRT continuous renal replacement therapy, eGFR estimated glomerular filtration rate, ICU LOS intensive care unit length of stay, calculated from the start of study antibiotic until ICU discharge, PDT pharmacodynamic target, SOFA Sequential Organ Failure Assessment

a Negative binomial regression model

${ }^{\mathrm{b}}$ Binary logistic regression model

that target attainment is observed in only about $60-65 \%$ of critically ill patients receiving antibiotics and considering the increasing resistance to antibiotics worldwide, higher dosing in these patients could be an alternative strategy to obtain better target attainment when TDM is not available. Carrie et al. showed that, in critically ill patients with ARC, higher than licensed dosing regimens of beta-lactam antibiotics may be safe and effective in reducing the rate of therapeutic failure [47]. Moreover, Imani et al. found that prescribed daily antibiotic dose $\geq$ 1.5 times the product information recommendations was associated with better target attainment [26]. Toxicity concerns as a result of drug accumulation are valid, but less pertinent given that toxicity thresholds are high for beta-lactam antibiotic agents [23]. However, serious adverse drug reactions related to excessively high serum levels have recently been reported, which further underscores a potential added value of TDM in critically ill patients [48-51]. Furthermore, to avoid high (peak) serum levels of beta-lactam antibiotics, prolonged or continuous infusion is an alternative dosing strategy to maximize target achievement and is likely to improve clinical outcomes in critically ill patients [52].

The current study has some limitations that should be noted. First, we have measured total drug concentrations with correction for protein binding based on the literature. Measuring unbound concentrations is desirable in critically ill patients, since the ratio of bound and unbound drugs can be subjected to changes because of disease characteristics of these patients. However, with the exception of ceftriaxone, the protein binding of most antibiotics in this study is too low to be clinically affected by, for example, a decreased serum albumin. Moreover, we analyzed unbound concentration of ceftriaxone in another cohort of critically ill patients [46] to support the clinical feasibility of calculating unbound fractions using an average PPB value. The mean fraction of ceftriaxone unbound plasma concentrations $(n=34)$ in the range of $0.05-40 \mathrm{mg} / \mathrm{L}$ was $12.3 \%$ [IQR $8.5-20$ ] (unpublished observations), which is comparable to the calculated unbound concentration used in this study and previously published data [31]. Second, MIC values were assumed from population estimates (ECOFF values) to calculate target attainment. Due to this approach, there is a chance that target attainment is underestimated in our study. Furthermore, the use of a measured MIC obtained by a single MIC determination is debatable, since routine clinical laboratories cannot determine MICs with sufficient accuracy due to the inherent assay variation in the MIC test and the variation in any MIC determination [53]. Although the ECOFF is in many situations similar to the clinical breakpoint, it is still important to closely evaluate the PK/PD target against the local drug resistance epidemiology. Third, we were not able to perform direct urinary creatinine measurements based on 24-h urine collections. We estimated serum creatinine clearance using the CKD-EPI Creatinine Equation formula, which is not validated for critically ill patients. 
However, it is unlikely that the study population with an eGFR $\geq 90 \mathrm{~mL} / \mathrm{min} / 1.73 \mathrm{~m}^{2}$ would in fact have had impaired renal function if calculated with urinary creatinine. Thus, we assume that possible misclassification of creatinine clearance did not invalidate our main observations. Fourth, we are aware that, in our regression models, baseline characteristics and target attainment were measured at two time points in the study period, respectively at inclusion and day of sampling. However, it is important to include some control variables that reflect the baseline characteristics of the patients. Finally, in the study population, cephalosporins, in particular cefotaxime, were overrepresented compared with the other classes of betalactam antibiotics. Target attainment and associated risk factors of the different antimicrobial agents in this context thus remain to be determined by more specific studies.

\section{Conclusions}

This study provides additional PDT attainment data and risk factors associated with target non-attainment to support beta-lactam antibiotic dosing in critically ill patients. Traditional beta-lactam antibiotic dosing results in low target attainment, less than two-thirds of our study population achieved the $100 \% f \mathrm{~T}>\mathrm{MIC}_{\mathrm{ECOFF}}$ target and only one-third the $100 \% f \mathrm{~T}>4 \times \mathrm{MIC}_{\mathrm{ECOFF}}$ target. Target attainment during beta-lactam therapy in critically ill patients may be anticipated at the bedside using predictor variables. Male patients with apparently normal or increased renal function and the use of CRRT were strong predictors of beta-lactam antibiotic exposure. Our data suggest that patients with eGFR $\geq 90 \mathrm{~mL} / \mathrm{min} / 1.73 \mathrm{~m}^{2}$ are at risk of target non-attainment, and this is associated with increased ICU LOS. Based on our data, we recommend TDM, selectively applied based on the described risk factors, during the early stages of beta-lactam therapy in critically ill patients. Whether these patients would benefit from more individualized dosing regimens should be evaluated by randomized controlled studies.

\section{Supplementary information}

Supplementary information accompanies this paper at https://doi.org/10. 1186/s13054-020-03272-z.

Additional file 1: Figure S1. Box and whisker plots of unbound peak plasma concentrations observed in critically ill patients treated with six beta-lactam antibiotics.

Additional file 2: Table S1. Characteristics of pooled antibiotic dosing and pharmacokinetic/pharmacodynamic (PK/PD) data.

\footnotetext{
Abbreviations

APACHE: Acute Physiology and Chronic Health Evaluation; ARC: Augmented renal clearance; $C L$ : Clearance; $C_{\text {max }}$ : Maximum concentration; $C_{\text {min }}$ : Minimum concentration; $\mathrm{CrCl}$ : Creatinine clearance; $\mathrm{Cl}$ : Continuous infusion; $\mathrm{CRP}$ : Creactive protein; CRRT: Continuous renal replacement therapy; ECOFF: Epidemiologic cut-off values; eGFR: Estimated glomerular filtration rate; EUCAST: European Committee on Antimicrobial Susceptibility Testing; El: Extended infusion; IM: Intermittent; ICU: Intensive care unit;
}

IQR: Interquartile range; LOS: Length of stay; MIC: Minimal inhibitory concentration; PD: Pharmacodynamic; PDT: Pharmacodynamic target; PI: Product information; PK: Pharmacokinetic; PK/PD: Pharmacokinetic/ pharmacodynamic; PPB: Plasma protein binding; SOFA: Sequential Organ Failure Assessment; TDM: Therapeutic drug monitoring; $f \mathrm{~T}>\mathrm{MIC}$ : Time for which the unbound drug concentration remains above the minimum inhibitory concentration during a dosing interval; UPLC-MS/MS: Ultraperformance liquid chromatography/tandem mass spectrometry; Vd: Volume of distribution

\section{Acknowledgements}

The authors are grateful to Prof. Dr. Johan W. Mouton for all his work in initiating this work. We thank Mujtaba Al Hadi, Rachida Malloul, Naoual Salhi, and Omar Rogouti for their help with the data collection and Dr. Wim J. R. Rietdijk for his contribution to the statistical analysis.

\section{Authors' contributions}

$\mathrm{AA}, \mathrm{NH}, \mathrm{TVG}$, and BK contributed to the conception and design of the study protocol. $A A, A D$, and $B K$ participated in the coordination of the study and data collection. AA and TE performed the pharmacokinetic analyses, and all authors contributed to the interpretation of the data. SB conducted the blood sample analysis. AA wrote the first draft of the manuscript. All authors contributed to subsequent drafts and gave final approval of the version to be published.

\section{Funding}

This project has received funding from the Erasmus University Medical Center MRace Grant. The Erasmus Medical Center approved the design of the trial, but had no role in the collection, analysis, and interpretation of data or in the writing manuscript or the decision to publish.

Ethics approval and consent to participate

The study was conducted in accordance with the principles of the Declaration of Helsinki. Approval for the study protocol (MEC-2015-502 / NL53551.078.15) was obtained from the Medical Ethics Committee of the Erasmus Medical Centre, Rotterdam, the Netherlands. Informed consent was obtained from the patients or their legal representative.

\section{Consent for publication}

Not applicable.

\section{Competing interests}

The authors declare that they have no competing interests.

\section{Author details}

${ }^{1}$ Department of Hospital Pharmacy, Erasmus University Medical Center, P.O. Box 2040, 3000 CA Rotterdam, the Netherlands. ${ }^{2}$ Department of Intensive Care, Maasstad Hospital, Rotterdam, The Netherlands. ${ }^{3}$ Department of Intensive Care, Erasmus University Medical Center, Rotterdam, The Netherlands. ${ }^{4}$ Department of Medical Microbiology and Infectious Diseases, Erasmus University Medical Center, Rotterdam, The Netherlands. ${ }^{5}$ Department of Medical Microbiology, Haaglanden Medical Center, The Hague, The Netherlands. 'Department of Clinical Pharmacy \& Toxicology, Leiden University Medical Center, Leiden, The Netherlands.

Received: 27 May 2020 Accepted: 2 September 2020 Published online: 15 September 2020

\section{References}

1. Vincent J-L, Sakr Y, Sprung CL, Ranieri VM, Reinhart K, Gerlach H, et al. Sepsis in European intensive care units: results of the SOAP study*. Crit Care Med. 2006;34(2):344-53.

2. Vincent JL, Rello J, Marshall J, Silva E, Anzueto A, Martin CD, et al. International study of the prevalence and outcomes of infection in intensive care units. JAMA. 2009;302(21):2323-9.

3. Bloos F, Rüddel H, Thomas-Rüddel D, Schwarzkopf D, Pausch C, Harbarth $S$, et al. Effect of a multifaceted educational intervention for anti-infectious measures on sepsis mortality: a cluster randomized trial. Intensive Care Med. 2017;43(11):1602-12 
4. Iregui M, Ward S, Sherman G, Fraser VJ, Kollef MH. Clinical importance of delays in the initiation of appropriate antibiotic treatment for ventilatorassociated pneumonia. Chest. 2002;122(1):262-8.

5. Leone M, Bourgoin A, Cambon S, Dubuc M, Albanèse J, Martin C. Empirical antimicrobial therapy of septic shock patients: adequacy and impact on the outcome*. Crit Care Med. 2003;31(2):462-7.

6. Pea F, Viale P, Furlanut M. Antimicrobial therapy in critically ill patients: a review of pathophysiological conditions responsible for altered disposition and pharmacokinetic variability. Clin Pharmacokinet. 2005; 44(10):1009-34.

7. Roberts JA, Abdul-Aziz MH, Lipman J, Mouton JW, Vinks AA, Felton TW, et al. Individualised antibiotic dosing for patients who are critically ill: challenges and potential solutions. Lancet Infect Dis. 2014;14(6):498-509.

8. Leligdowicz A, Matthay MA. Heterogeneity in sepsis: new biological evidence with clinical applications. Crit Care. 2019;23(1):80.

9. Craig WA. Interrelationship between pharmacokinetics and pharmacodynamics in determining dosage regimens for broad-spectrum cephalosporins. Diagn Microbiol Infect Dis. 1995;22(1):89-96.

10. Craig WA. Pharmacokinetic/pharmacodynamic parameters: rationale for antibacterial dosing of mice and men. Clin Infect Dis. 1998;26(1):1-12.

11. Turnidge JD. The pharmacodynamics of $\beta$-lactams. Clin Infect Dis. 1998; 27(1):10-22

12. McKinnon PS, Paladino JA, Schentag JJ. Evaluation of area under the inhibitory curve (AUIC) and time above the minimum inhibitory concentration ( $\mathrm{T}>\mathrm{MIC}$ ) as predictors of outcome for cefepime and ceftazidime in serious bacterial infections. Int J Antimicrob Agents. 2008; 31(4):345-51.

13. Abdul-Aziz MH, Lipman J, Mouton JW, Hope WW, Roberts JA. Applying pharmacokinetic/pharmacodynamic principles in critically ill patients: optimizing efficacy and reducing resistance development. Semin Respir Crit Care Med. 2015;36(1):136-53.

14. Huttner A, Von Dach E, Renzoni A, Huttner BD, Affaticati M, Pagani $L$, et al. Augmented renal clearance, low $\beta$-lactam concentrations and clinical outcomes in the critically ill: an observational prospective cohort study. Int J Antimicrob Agents. 2015;45(4):385-92.

15. Li C, Du X, Kuti JL, Nicolau DP. Clinical pharmacodynamics of meropenem in patients with lower respiratory tract infections. Antimicrob Agents Chemother. 2007;51(5):1725-30

16. Wong G, Brinkman A, Benefield RJ, Carlier M, De Waele JJ, El Helali N, et al. An international, multicentre survey of $\beta$-lactam antibiotic therapeutic drug monitoring practice in intensive care units. J Antimicrob Chemother. 2014; 69(5):1416-23.

17. Tam VH, McKinnon PS, Akins RL, Rybak MJ, Drusano GL. Pharmacodynamics of cefepime in patients with Gram-negative infections. J Antimicrob Chemother. 2002;50(3):425-8.

18. Aitken SL, Altshuler J, Guervil DJ, Hirsch EB, Ostrosky-Zeichner LL, Ericsson $C D$, et al. Cefepime free minimum concentration to minimum inhibitory concentration ( $\mathrm{fCmin} / \mathrm{MIC}$ ) ratio predicts clinical failure in patients with Gram-negative bacterial pneumonia. Int J Antimicrob Agents. 2015;45(5): 541-4.

19. De Waele JJ, Carrette S, Carlier M, Stove V, Boelens J, Claeys G, et al. Therapeutic drug monitoring-based dose optimisation of piperacillin and meropenem: a randomised controlled trial. Intensive Care Med. 2014;40(3): 380-7.

20. Wheat PF. History and development of antimicrobial susceptibility testing methodology. J Antimicrob Chemother. 2001;48(suppl_1):1-4.

21. Roberts JA, Paul SK, Akova M, Bassetti M, De Waele JJ, Dimopoulos G, et al DALI: defining antibiotic levels in intensive care unit patients: are current ßlactam antibiotic doses sufficient for critically ill patients? Clin Infect Dis. 2014;58(8):1072-83.

22. Taccone FS, Laterre P-F, Dugernier T, Spapen H, Delattre I, Wittebole $X$, et al. Insufficient $\beta$-lactam concentrations in the early phase of severe sepsis and septic shock. Critical Care. 2010;14(4):R126-R.

23. Imani S, Buscher H, Marriott D, Gentili S, Sandaradura I. Too much of a good thing: a retrospective study of $\beta$-lactam concentration-toxicity relationships. J Antimicrob Chemother. 2017;72(10):2891-7.

24. Carrié C, Petit L, d'Houdain N, Sauvage N, Cottenceau V, Lafitte M, et al. Association between augmented renal clearance, antibiotic exposure and clinical outcome in critically ill septic patients receiving high doses of $\beta$ lactams administered by continuous infusion: a prospective observational study. Int J Antimicrob Agents. 2018;51(3):443-9.
25. De Waele JJ, Lipman J, Akova M, Bassetti M, Dimopoulos G, Kaukonen M, et al. Risk factors for target non-attainment during empirical treatment with $\beta$-lactam antibiotics in critically ill patients. Intensive Care Med. 2014;40(9): 1340-51.

26. Imani S, Buscher H, Day R, Gentili S, Jones GRD, Marriott D, et al. An evaluation of risk factors to predict target concentration non-attainment in critically ill patients prior to empiric $\beta$-lactam therapy. Eur J Clin Microbiol Infect Dis. 2018;37(11):2171-5.

27. Woksepp H, Hällgren A, Borgström S, Kullberg F, Wimmerstedt A, Oscarsson $A$, et al. High target attainment for $\beta$-lactam antibiotics in intensive care unit patients when actual minimum inhibitory concentrations are applied. Eur J Clin Microbiol Infect Dis. 2017;36(3):553-63.

28. Julious SA, Patterson SD. Sample sizes for estimation in clinical research. Pharm Stat. 2004;3(3):213-5.

29. Abdulla A, Bahmany S, Wijma RA, van der Nagel BCH, Koch BCP. Simultaneous determination of nine $\beta$-lactam antibiotics in human plasma by an ultrafast hydrophilic-interaction chromatography-tandem mass spectrometry. J Chromatogr B. 2017;1060:138-43.

30. (FDA) USFaDA. Guidance for industry: bioanalytical method validation 2013. Available from: https://www.fda.gov/downloads/drugs/guidances/ucm3681 07.pdf.

31. Ulldemolins M, Roberts JA, Rello J, Paterson DL, Lipman J. The effects of hypoalbuminaemia on optimizing antibacterial dosing in critically ill patients. Clin Pharmacokinet. 2011;50(2):99-110.

32. Zhang Y, Huo M, Zhou J, Xie S. PKSolver: an add-in program for pharmacokinetic and pharmacodynamic data analysis in Microsoft Excel. Comput Methods Prog Biomed. 2010;99(3):306-14.

33. EUCAST. Clinical breakpoints and dosing of antibiotics: EUCAST; 2019 [updated 08-01-2019]. Available from: http://www.eucast.org/clinical_ breakpoints/.

34. Mouton JW, Vinks AA. Pharmacokinetic/pharmacodynamic modelling of antibacterials in vitro and in vivo using bacterial growth and kill kinetics: the minimum inhibitory concentration versus stationary concentration. Clin Pharmacokinet. 2005:44(2):201-10.

35. Delattre IK, Taccone FS, Jacobs F, Hites M, Dugernier T, Spapen H, et al. Optimizing $\beta$-lactams treatment in critically-ill patients using pharmacokinetics/pharmacodynamics targets: are first conventional doses effective? Expert Rev Anti-Infect Ther. 2017;15(7):677-88.

36. Tam VH, Chang KT, Zhou J, Ledesma KR, Phe K, Gao S, et al. Determining $\beta$ lactam exposure threshold to suppress resistance development in Gramnegative bacteria. J Antimicrob Chemother. 2017;72(5):1421-8.

37. Soldin OP, Mattison DR. Sex differences in pharmacokinetics and pharmacodynamics. Clin Pharmacokinet. 2009;48(3):143-57.

38. Udy AA, Baptista JP, Lim NL, Joynt GM, Jarrett $P$, Wockner $L$, et al. Augmented renal clearance in the ICU: results of a multicenter observational study of renal function in critically ill patients with normal plasma creatinine concentrations*. Crit Care Med. 2014:42(3):520-7.

39. Claus BOM, Hoste EA, Colpaert K, Robays H, Decruyenaere J, De Waele JJ. Augmented renal clearance is a common finding with worse clinical outcome in critically ill patients receiving antimicrobial therapy. J Crit Care. 2013;28(5):695-700.

40. Arabi Y, Venkatesh S, Haddad S, Shimemeri AAL, Malik SAL. A prospective study of prolonged stay in the intensive care unit: predictors and impact on resource utilization. Int J Qual Health Care. 2002;14(5):403-10.

41. Roberts JA. Using PK/PD to optimize antibiotic dosing for critically ill patients. Curr Pharm Biotechnol. 2011;12(12):2070-9.

42. Roberts JA, Hope WW, Lipman J. Therapeutic drug monitoring of $\beta$-lactams for critically ill patients: unwarranted or essential? Int J Antimicrob Agents. 2010:35(5):419-20.

43. Sime F, Roberts MS, Peake SL, Lipman J, Roberts JA. Does beta-lactam pharmacokinetic variability in critically ill patients justify therapeutic drug monitoring? A systematic review. Ann Intensive Care. 2012;2(1):35.

44. Charmillon A, Novy E, Agrinier N, Leone M, Kimmoun A, Levy B, et al. The ANTIBIOPERF study: a nationwide cross-sectional survey about practices for $\beta$-lactam administration and therapeutic drug monitoring among critically ill patients in France. Clin Microbiol Infect. 2016;22(7):625-31.

45. Carlier M, Stove V, Wallis SC, De Waele JJ, Verstraete AG, Lipman J, et al. Assays for therapeutic drug monitoring of $\beta$-lactam antibiotics: a structured review. Int J Antimicrob Agents. 2015;46(4):367-75.

46. Abdulla A, Ewoldt TMJ, Hunfeld NGM, Muller AE, Rietdijk WJR, Polinder S, et al. The effect of therapeutic drug monitoring of beta-lactam and 
fluoroquinolones on clinical outcome in critically ill patients: the DOLPHIN trial protocol of a multi-centre randomised controlled trial. BMC Infect Dis. 2020;20(1):57.

47. Carrié C, Chadefaux G, Sauvage N, de Courson H, Petit L, Nouette-Gaulain K, et al. Increased $\beta$-lactams dosing regimens improve clinical outcome in critically ill patients with augmented renal clearance treated for a first episode of hospital or ventilator-acquired pneumonia: a before and after study. Crit Care. 2019;23(1):379.

48. Lamoth F, Buclin T, Pascual A, Vora S, Bolay S, Decosterd LA, et al. High cefepime plasma concentrations and neurological toxicity in febrile neutropenic patients with mild impairment of renal function. Antimicrob Agents Chemother. 2010;54(10):4360-7.

49. Beumier M, Casu GS, Hites M, Wolff F, Cotton F, Vincent $\mathrm{J}$, et al. Elevated $\beta$ lactam concentrations associated with neurological deterioration in ICU septic patients. Minerva Anestesiol. 2015;81(5):497-506.

50. Huwyler T, Lenggenhager L, Abbas M, Ing Lorenzini K, Hughes S, Huttner B, et al. Cefepime plasma concentrations and clinical toxicity: a retrospective cohort study. Clin Microbiol Infect. 2017;23(7):454-9.

51. Le Turnier P, Navas D, Garot D, Guimard T, Bernard L, Tattevin P, et al. Tolerability of high-dose ceftriaxone in CNS infections: a prospective multicentre cohort study. J Antimicrob Chemother. 2019;74(4):1078-85.

52. Vardakas KZ, Voulgaris GL, Maliaros A, Samonis G, Falagas ME. Prolonged versus short-term intravenous infusion of antipseudomonal $\beta$-lactams for patients with sepsis: a systematic review and meta-analysis of randomised trials. Lancet Infect Dis. 2018;18(1):108-20.

53. Mouton JW, Muller AE, Canton R, Giske CG, Kahlmeter G, Turnidge J. MICbased dose adjustment: facts and fables. J Antimicrob Chemother. 2017; 73(3):564-8

\section{Publisher's Note}

Springer Nature remains neutral with regard to jurisdictional claims in published maps and institutional affiliations.

Ready to submit your research? Choose BMC and benefit from:

- fast, convenient online submission

- thorough peer review by experienced researchers in your field

- rapid publication on acceptance

- support for research data, including large and complex data types

- gold Open Access which fosters wider collaboration and increased citations

- maximum visibility for your research: over $100 \mathrm{M}$ website views per year

At $\mathrm{BMC}$, research is always in progress.

Learn more biomedcentral.com/submissions 